\title{
Size Effects in Agent-Based Macroeconomic Models: An Initial Investigation
}

\author{
Shu-Heng Chen ${ }^{1}$, Ying-Fang Kao ${ }^{1}$, Bin-Tzong Chie ${ }^{2}$, Timo Meyer ${ }^{3}$, and \\ Ragupathy Venkatachalam ${ }^{1,4}$ \\ 1 AI-ECON Research Center, Department of Economics, National Chengchi University, \\ Taipei, Taiwan 11605. chen. shuheng@gmail.com \\ 2 Department of Industrial Economics, Tamkang University, Tamshui, New Taipei City, \\ Taiwan 251. \\ 3 Department of Physics, Georg-August University Göttingen, 37073 Göttingen, \\ Germany. \\ 4 Institute of Management Studies, Goldsmiths, University of London, New Cross, \\ London, SE14 6NW, UK
}

\begin{abstract}
We investigate the scale-free property of an agent-based macroeconomic model initially proposed by Wright (2005), called the Social Architecture (SA) model. The SA model has been shown to be able to replicate a number of important features of a macroeconomy, such as patterns concerning economic growth, business cycles, industrial dynamics and income distribution. We explore whether macroeconomic stylized features resulting from this model are robust when the number of agents populating the (model) economy vary. We simulate the model by systematically varying the agent population with 100, 500, 1000, 2,000, 4,000, 8,000 and 10,000 agents. Our results indicate that the SA model does exhibit significant size effects for several important variables.
\end{abstract}

Keywords: Maximum Entropy Principle, Size Effect, Agent-Based Macroeconomic Model, Circular Flow

\section{Motivation and Introduction}

One of the greatest potential contributions that ACE could make to macroeconomic theory is permitting the constructive exploration of scale effects without the external imposition of artificial coordination devices. What does it matter if an economy has 10,000 versus 300 million participants? What macroeconomic purposes are served by small-scale models, and which require a scale closer to empirical reality? Do macroeconomies exhibit important regularities that simply cannot be generated using small scale models? ([10], p. 248; Italics added.)

Among the many agent-based macroeconomic models, Ian Wright's Social Architecture $(S A)$ model $([13,14,8])$ is unique in its model-design principle. Wright 
models the circular flow of the macroeconomy in an agent-based fashion, i.e., by individualizing each economic action that contributes to the flow, and then randomizing each action using the entropy-maximization principle (the EM principle). ${ }^{1}$ With this design, no further behavioral considerations are given to the consumers, firms, employees and employers. This is in accordance with the EM principle, which argues for allowing a maximum amount of uncertainty, given current knowledge (or the lack of it) regarding a phenomenon. While the EM principle has been applied in other agent-based economic models, such as double auction markets $([7,12])$ and financial markets $([6,4])$, to the best of our knowledge, there is no application of this principle in the literature on agentbased macroeconomic models, with the exception of Wright's model. ${ }^{2}$

Economists often have reservations concerning the use of the EM principle. However, the fundamental concern for the proponents of the EM principle is not whether agents behave randomly in reality, but rather the degree of empirical relevance of the given model. On this point, Wright makes a strong case for the SA model by showing that it is able to replicate several stylized facts, specifically on the distribution of a number of key economic variables, such as firms' size and demise, the duration of recessions, and the distribution of income and wealth. Perhaps the most impressive feature of this model is that it comprises just three parameters.

Given its 'initial success', we would like to examine whether this model is size-free. Here, the 'size' of the model refers to the number of agents in the model. According to $[13,14]$, the number of agents is merely a scaling parameter and hence it should not affect the relative dynamics since the computational rules do not refer to them. This parameter is fixed at 1,000 throughout Wright's entire simulation. It is legitimate to wonder whether the fundamental results obtained in his analysis, such as the Zipf law concerning the firm size, would be invariant to changes in the number of agents. We ask the following questions: Are the stable patterns (distributions) discussed by Wright so fundamental that size plays no role in the model? If size does matter, then what properties are sensitive to size?

The significance of this study is three-fold. First, size, as characterized by the number of agents, has been shown to be a crucial parameter in many agentbased models $([5,2,11])$, where finite size effects are observed. Therefore, the results obtained using a fixed size should be carefully evaluated if they are not size-free. Second, in the context of agent-based models, it is also interesting to ask when and why size matters. Normally, size can matter because the aggregation is not merely a linear scaling-up in most agent-based settings. Interactions can alter some economic relations when size changes. Hence, by studying the

\footnotetext{
1 The EM principle is also known as the zero-intelligence agent in the literature. However, as [3] has argued, this term may be misleading since the behavioral assumption may have nothing to do with the cognitive abilities of agents. Therefore, we prefer using a different and a more formal term.

2 For a survey of the use of the entropy-maximization principle in the agent-based modeling of economics and finance, the interested reader is referred to [9].
} 
size effect, we can also gain further insights into the interaction schemes employed in the model. Third, size may also matter in the real-world: for instance, the behavior of small economies can be fundamentally different from that of large economies due to size sensitivity. If so, it is desirable to identify stable, scale-independent macroeconomic properties, which can help facilitate meaningful discussions about experimental policy interventions in agent-based environments. More importantly, they can help to choose a canonical model from different candidate explanations.

The rest of the paper is organized as follows. Section 2 provides a brief overview of the Wright model, mainly, [13]. Section 3 articulates the design of the simulation, including the time structure (the time flow) of the simulation run in this paper and the parameters applied in this paper. Section 4 presents the simulation results ${ }^{3}$ as well as an examination of the size effect, followed by a discussion and conclusion in Section 5.

\section{An Overview of the Wright Model}

\subsection{The Modeling Principle}

In the Wright model, all micro decisions are essentially random, and agents' behavior is modeled based on the EM principle. The model can be regarded as an extension of the Gode-Sunder model in the double auction market ([7]) to a circular-flow macroeconomic model. In the Gode-Sunder model, each agent needs to make only one decision at a time, either to bid or to ask, and the EM principle is applied to this single decision. Whereas in the circular-flow model agents need to make a few more decisions along the flow, and the EM principle is applied to all these decisions.

Figure 1 outlines a simple circular flow. There are two types of agents, households and firms and there are two markets, a labor market and a goods market. There is neither a public sector nor a financial sector; hence the financial market does not exist in this simple circular flow. The household sector is composed of $N$ agents ( $N$ single-head households). These $N$ agents also constitute the labor market as employers (firms, the single-owner firms), employees and some who are unemployed. The products will be sold to households in the goods market. There are basically two flows that run in the reverse direction. The inside circle represents the flow of labor, products and real consumption, and the outside circle depicts the flow of wage payments, expenditures, and firm revenues.

The circular flow can be completed in many different ways. Here, we follow Wright's sequence, i.e.,

$$
\text { goods market } \rightarrow \text { labor market } \rightarrow \text { goods market. }
$$

We begin with the goods market, and there is money flowing from households (expenditures) to firms (revenues). We then end up with the labor market, and

\footnotetext{
3 The simulations are performed using both Matlab and NetLogo, and the codes are publicly available on the Internet (see Section 3)
} 


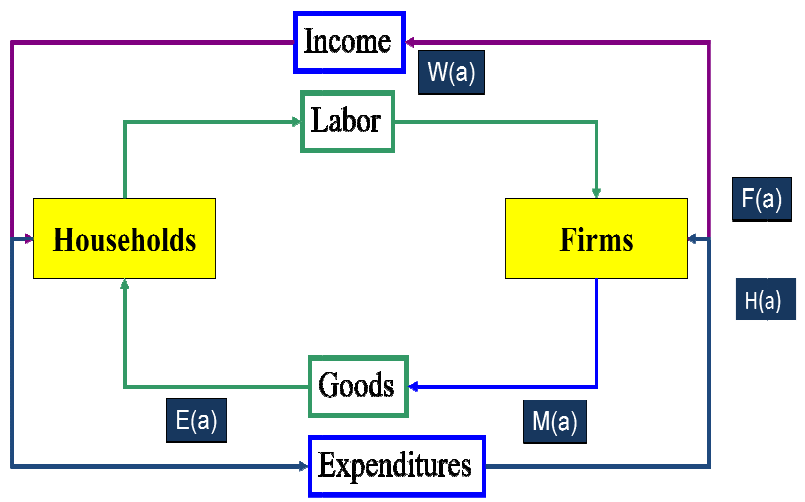

Fig. 1: Circular Flow of the Economy

there is a flow of money from firms (revenues) to households (wages). To be brief, we call this sequence $G M \rightarrow L M \rightarrow G M$.

However, from the flow as shown above, it is not clear who can buy from whom and who can hire whom. For this purpose, we incorporate a labor-market mechanism that matches at the beginning of the flow, i.e.,

$$
\text { L Matches } \rightarrow \mathrm{GM} \rightarrow \mathrm{LM} \rightarrow \mathrm{GM} \text {. }
$$

The decisions involved over the entire flow include where to work, whether to start (close) a firm, how much to consume, where to consume, how many employees to hire (fire), whom to hire, and what wages to pay. There are a maximum of nine decisions in one single flow. Of course, not all of them will be encountered in a single run; different agents may encounter different decision problems, depending on each agent's status as an employer, employee or simply unemployed. The upshot is that, except for the closing, the firing, and the hiring decisions that are deterministic and which are dependent on the firm's working capital, the other six decisions are all based on the EM principle.

\subsection{Agents and Network}

We shall begin with a labor market network. We follow the notations frequently used in the network literature. Let $(i, j)$ be a link connecting agents $i$ and $j$, indicating that agent $i$ is hired by agent $j$. Since $(i, j)$ is not the same as $(j, i)$, the labor market network considered here is directed. Let $g(t)$ be the labor market network at time $t$, which is a collection of all employee-employer relations.

$$
g(t)=\{(i, j): i, j \in \mathbf{N}, i \neq j\},
$$

where $\mathbf{N}$ is the set of all agents, and the cardinality of $\mathbf{N}$ is $N$.

Then the agent $i$ who has a connection in the labor market can be either an employee or an employer and agent $i$ who has no connection in the labor 
market is considered to be unemployed. Equation (1) already excludes the case of being self-employed, but we further assume that each agent can have at most one job, i.e., one employer. We also exclude the possibility each agent can be an employee and an employer simultaneously; hence, for agent $i$,

$$
\text { if } \exists j,(j, i) \in g(t) \text {, then } \nexists k, \ni(i, k) \in g(t) .
$$

In other words, the network can be viewed as a bipartite graph at any given time. ${ }^{4}$ Each employer can, however, have many employees, and the set of his employees (connections) at time $t, g_{i}(t)$ is denoted by

$$
g_{i}(t)=\{(j, i):(j, i) \in g(t)\} .
$$

As for agent $i$ who is not an employer, he is either an employee, i.e., $\exists j,(i, j) \in$ $g(t)$, or unemployed, $g_{i}(t)=\varnothing$. To simplify these expressions, we use $g_{i}(t)=$ $\{j\}\left(j \in g_{i}(t)\right)$ if agent $i$ is an employer at time $t$, similarly $g_{i}(t)=j$ if agent $i$ is an employee, and $g_{i}(t)=0$, if agent $i$ is unemployed at time $t$. The collection of employers (firms), employees, and the unemployed at time $t$ will be denoted by $F(t), L(t)$, and $U(t)$. Clearly, $F(t) \cup L(t) \cup U(t)=\mathbf{N}$, and $F(t) \cap L(t) \cap U(t)=\varnothing$.

\subsection{Behavioral Rules in the Flow of the Economy}

The economy proceeds in a way that is parallel to the circular flow, shown in Figure 1 . We run this cycle for many iterations. At time $t$, we begin by randomly selecting an agent from the set of agents $\mathbf{N}$, and call him agent $a$. Agent $a$ can be an employer $(a \in F(t-1))$, an employee $(a \in L(t-1))$, or unemployed ( $a \in$ $U(t-1)$ ). Regardless of his current status, agent $a$ will go through the economy along the sequence

$$
\text { L Matches } \rightarrow \mathrm{GM} \rightarrow \mathrm{LM}
$$

and make decisions for those circumstances which fit his status.

Labor Market Matches The sequence starts with the labor market matching. If $a \in U(t-1)$, then the following action applies. Agent $a$ enters the labor market and is randomly matched to an agent $j(j \in F(t-1) \cup U(t-1), j \neq a)$ with the following probability:

$$
p((a, j))(t)=\frac{m_{j}(t-1)}{\sum_{k \in F(t-1) \cup U(t-1) /\{a\}} m_{k}(t-1)},
$$

where $m_{i}(t)$ is the money holding of agent $i$ at time $t$. Equation (3) implies that it is more likely for the unemployed agent $a$ to find a job in firms which are economically more successful and have accumulated more working capital $\left(m_{k}\right)$. This is similar to the preferential attachment mechanism used to generate the

\footnotetext{
${ }^{4}$ However, since the status of being an employee or an employer can change over time, a directed graph is more convenient.
} 
scale-free network ([1]). Notice that Equation (3) does not exclude the possibility that an agent $a$ can be recruited by another unemployed agent $j$. In the latter case, a new firm is formed. The search (match) is one-shot, and its success is primarily determined by the affordability of agent $j$, i.e., Equation (4).

$$
g_{a}(t)=\left\{\begin{array}{l}
j, \text { if } m_{j}>\bar{w}, \\
0, \text { otherwise. }
\end{array}\right.
$$

where $\bar{w}$ is a reference wage of the labor market, to be detailed in Equation (13).

Goods Market: Expenditures After the labor market matching, the flow proceeds to the goods market. An agent $j$ randomly selected from $\mathbf{N} /\{a\}$ enters the goods market. The total consumption of agent $j$ at time $t, C_{j}(t)$, is randomly determined, following a uniform distribution.

$$
C_{j}(t) \sim U\left[0, m_{j}(t-1)\right]
$$

His consumption $C_{j}(t)$ will be attributed to the market value of products. Let $V(t)$ be the pool of the market value which has been accumulated up to $t-1$, i.e., the market value which has not been distributed. Then

$$
V(t)=V(t-1)+C_{j}(t) .
$$

Goods Market: Revenues From the circular flow (Figure 1), the money in the market value (expenditure) pool, $V(t)$, will be distributed to the firms. This distribution mechanism is also random and is inclined towards the big firm. First, if agent $a$ is not unemployed $(a \notin U(t))$, revenue is uniformly sampled from the market value pool, i.e.,

$$
R(t) \sim U[0, V(t)]
$$

Second, this revenue will be attributed to a firm in the following manner.

$$
\left\{\begin{array}{c}
m_{a}(t)=m_{a}(t-1)+R(t), \text { if } a \in F(t), \\
m_{g_{a}(t)}(t)=m_{g_{a}(t)}(t-1)+R(t), \text { if } a \in L(t) .
\end{array}\right.
$$

After the money transfer, the pool of market value will also updated accordingly.

$$
V(t) \leftarrow V(t)-R(t)
$$

Labor Market: Employment and Wages As per the circular flow, after the market value has been distributed to firms as their revenues, the transfer of this revenue to labor income or wages follows. If the randomly selected actor $a$ is an employer $(a \in F(t-1))$, then he has to make two decisions in this stage: on employment and wages. The employment decision concerns the adjustment of the labor demand. The employer $a$ will decide the number of workers he can afford, based on the reference wage $\bar{w}$ and his current money holdings. Let $u_{a}(t)$ 
be the number of workers that employer $a$ has to lay off. Then the demand for labor is given by Equation (10).

$$
u_{a}(t)=\max \left(\left|g_{a}(t-1)\right|-\left\lfloor\frac{m_{a}(t)}{\bar{w}}\right\rfloor, 0\right),
$$

where $\left|g_{a}(t-1)\right|$ denotes the number of workers hired by employer $a$ at time $t-1 ;\lfloor x\rfloor$ is the greatest integer that is less than or equal to $x .\left\lfloor\frac{m_{a}(t)}{\bar{w}}\right\rfloor$, therefore, is the affordable demand for labor, and subtracting it from $\left|g_{a}(t-1)\right|$ gives the number of layoffs. The layoffs will be randomly selected from $g_{a}(t-1)$. Notice that the sup operator, max, is imposed; hence the labor demand can only be adjusted downward, and not upward. In the worst case, firm $a$ may fire all its workers. If that happens, the status of agent $a$ will change from that of an employer $\left(g_{a}(t-1)=\{j\}\right)$, to unemployed $\left(g_{a}(t)=0\right)$. The status of all those who are fired also needs to be updated as well. Let the set $U_{a}(t)$ be the set of agents who are fired by firm $a$, then

$$
g_{i}(t)=0, \forall i \in U_{a}(t) .
$$

After the employment decision, if

$$
g_{a}(t)=g_{a}(t-1) / U_{a}(t) \neq \varnothing,
$$

then the remaining workers, $i \in g_{a}(t)$, will be paid their wages according to the following random mechanism:

$$
w_{i}(t) \sim\left\{\begin{array}{l}
U\left[w_{L}, w_{H}\right], \text { if } m_{a}\left(t_{i}\right) \geq w_{H}, \\
U\left[0, m_{a}\left(t_{i}\right)\right], \text { otherwise, }
\end{array}\right.
$$

where $m_{a}\left(t_{i}\right)$ is the money holdings of firm $a$ upon the moment of paying wages to employee $i$, and $w_{L}$ and $w_{H}$ are the floor and the ceiling of the wage range for the employee. When firm $a$ 's money holdings are sufficient to cover the upper bound of this range, Equation (12) states that the wage will be randomly determined according to the uniform distribution $U\left[w_{L}, w_{H}\right]$. The reference wage $\bar{w}$ which we use in this paper is in fact the midpoint of this range, i.e.,

$$
\bar{w}=\frac{\left(w_{L}+w_{H}\right)}{2} .
$$

Nonetheless, upon the moment of paying wages to employee $i$, if employer $a$ finds that his money holdings are less than the threshold $w_{H}$, the wage will be uniformly randomly determined from 0 to the firm's maximum affordability, $m_{a}\left(t_{i}\right)$. After paying all wages to his employees, the money holdings of employer $a$ will be updated again as follows:

$$
m_{a}(t) \leftarrow m_{a}(t)-\sum_{i \in g_{a}(t)} w_{i}(t),
$$

and in the meantime

$$
m_{i}(t)=m_{i}(t-1)+w_{i}(t), \forall i \in g_{a}(t) .
$$


This then comes to the end of time $t$. For all other agents $i$ who are not involved in any part of the circular flow, either as an unemployed worker, an employer, an employee or a consumer, his money holding will remain unchanged, namely, $m_{i}(t-1)=m_{i}(t)$. The state of the economy at time $t, S(t)$, can be summarized as a collection of triplets:

$$
\left.S(t)=\left\{\left(m_{i}(t), g_{i}(t), w_{i}(t)\right)\right\}: 1 \leq i \leq N\right\}
$$

To sum up, Wright's agent-based model of the circular flow can be perceived as a 'relay race'. Each 'runner' (agent) is randomly sampled from $\mathbf{N}$ with replacement. When one runner, at time $t$, 'runs through' the flow, the next runner, at time $t+1$, takes the baton, and does the next run through. This cycle will be repeated for a sufficiently large number of times so that almost all agents have been introduced to the economy at least once (on average) within a given length of time, say, a day, a week, or a month. Then the clock moves to the next day, week, or month, and so on.

\section{Simulation Design}

In our simulation, we have running time in ticks (the tick data), loops of ticks (the monthly data), and loops of loops (yearly data). Each tick $t$ represents one iteration, which corresponds to the time interval $[t, t-1)$ and also to one round of the sequence L Matches $\rightarrow \mathrm{GM} \rightarrow \mathrm{LM}$. The iterations will be repeated $N$ (i.e., the number of agents) times and this together forms one loop (a duration of one month) of simulations. By setting the number of iterations as $N$, we ensure that each agent, on average, will be sampled once per month. The entire simulation is run for a number of 'years'. The notation $X\left(t_{m o}^{y r}\right)$ indicates the variable $A$ at the $t^{t h}$ iteration of the month mo of year $y r$. In this paper, $t=1, \ldots, N, m o=1, \ldots, 12$, $y r=1, \ldots, 100$. In this setting, our time scale and duration of the simulation is entirely consistent with [13].

In this article, instead of merely replicating Wright's model, we alter the size of the population, $N$, for the purpose of examining the possible size effects, in general. Therefore, in some cases we do not have an a priori assumption concerning distributions as Wright would have had. For example, we do not assume a priori that the GDP growth rate follows a Laplace distribution. Instead, we examine whether the distributions are sensitive to size. We consider $100,500,1,000,2,000,4,000,8,000,10,000$ agents (Table 1). To focus on the size effect alone, we keep the money holding per capita fixed throughout all simulations at 100 , which is independent of the size of population. ${ }^{5}$ We repeat the simulations 100 times for each size variation. We also compare the resulting distribution of these estimates using appropriate statistical tests. Table 1 gives a summary of the values of the control parameters used to run the simulation of this paper. The results reported in this paper are all obtained from MATLAB.

\footnotetext{
${ }^{5}$ Notice that, since the amount of money is initially randomly distributed among all agents, we can only fix the money holding per capita, but not the possible distribution effect.
} 
Table 1: Table of Control Parameters

\begin{tabular}{lll}
\hline$N \quad$ Number of agents & $100,500,1,000,2,000$, \\
& & $4,000,8,000,10,000$ \\
$\bar{m} \quad$ Average money holding & 100 \\
$w_{L}$ Wage (lower bound) & 10 \\
$w_{H}$ Wage (upper bound) & 90 \\
$Y r$ Number of years per simulation & 100 \\
\multicolumn{2}{l}{ Number of trials } & 100 \\
\hline
\end{tabular}

We collect monthly data on different variables during the course of the simulation and group them together in frequencies (monthly, yearly) along the same lines as Wright (2005). The variables (data) and their different frequencies are defined as follows.

- Monthly observations Monthly data are collected for the following variables: 1) Firm Size: Number of employees observed at each firm, at the end of each month. 2) Firm Demise (Ratio) ${ }^{6}$ : The number of firms that cease to exist during each month in the economy.

- Yearly observations Yearly data are collected for the following variables: 1) Percentage of Capitalists, Percentage of Workers, and Unemployment Rate

2) Growth of Firms: Size of sales and employment, 3) GDP Growth, 4) Wage and Profit Share, 5) Total Wealth, 6) Profit Rate,

- Long-run observations Duration of Recession: A recession is said to begin in $y r$ when the output expansion ceases and the economy begins to contract. The recession ends when the reverse happens.

Examining the distributions for each of these variables and their respective conformity with the observed stylized facts is in itself an interesting task. However, for this paper, we focus exclusively on the size effects of the SA model and not on their degree of conformity with actual stylized facts. We examine whether economies of different size $(N)$ will generate different distributions for each of the macroeconomic variables. Since the underlying data generating process as specified by the entropy-maximizing model is stochastic, to obtain statistical reliability for the simulation results, for each treatment we run trials 100 times (Table 1). Therefore, for each variable, we obtain 100 ensembles and our analysis of the size effect is then based on a comparison across these 100 ensembles.

Since we are comparing different distributions and variables with different frequencies, we need to choose a representative distribution for each treatment, so that we perform comparisons across treatments. Once we fix the relevant period that we wish to consider for each variable, we pool the data across repeated

\footnotetext{
${ }^{6}$ Since we compare distributions across different sizes of the economy, we normalize the absolute value of the number of firm demises into ratios with respect to the size of the economy.
} 
Table 2: Range of Data and Statistical Tests

\begin{tabular}{lccc}
\hline Variable & Data Type & Duration & Test \\
\hline Capitalist Ratio & Historical Means & All & Wilcoxon RS \\
Worker Ratio & Historical Means & All & Wilcoxon RS \\
Unemployment Ratio & Historical Means & All & Wilcoxon RS \\
Firm Demise Ratio & Historical Means & All & Wilcoxon RS \\
Wealth Gini & Historical Means & Last 10 years & Wilcoxon RS \\
Yearly log GDP growth & Pooled & Last 10 years (1200 obs) & K-S \\
Yearly Wage Rate & Pooled & Last 10 years (1200 obs) & K-S \\
FirmGrowth (Employment $)$ & Pooled & Last 10 years & K-S \\
FirmGrowth (Sales ) & Pooled & Last 10 years & K-S \\
Rate of Profit & Pooled & Last 10 years & K-S \\
Recession Yearly & Pooled & All & K-S \\
Firm-Size Dist & Pooled & Last Month & K-S \\
\hline Wilcoxon RS denotes the Wilcoxon Rank Sum test and K-S denotes the Kolmogorov-Smirnov test.
\end{tabular}

simulations (100 of them) for each treatment. For this purpose, we consider the last few periods for each variable under the assumption that their distributions stabilize over the course of the simulation ${ }^{7}$. Table 2 provides a summary of the variables, data type used, duration of the data and the statistical test employed. We then compare the representative distributions across treatments to examine the size effects. Although some of our results in the replications may not fully confirm with what Wright has demonstrated, especially with regard to replicating specific aggregate distributions, we believe that our simulation is faithful to his protocol. This exercise can, in its own right, be considered as an independent study that investigates the size effects in a Wright-like model.

\section{Simulation Results and Discussion}

From our simulation of the Wright model, we find that the resulting distributions of several macroeconomic variables seem to be size dependent. We use the Kolmogorov-Smirnov test, a non-parametric test, to compare different distributions across treatments. When we compare means across different distributions, we use the Wilcoxon rank sum test. The rank sum test is less sensitive to outliers compared to the two-sample t-test. Although our sample size is big enough to use the t-test, our data appear to have heterogeneous variances and therefore the Wilcoxon rank sum test is more appropriate.

Table 3 presents the results of the statistical tests that compare the distributions for different sizes. We use $1 \%$ as the significance level and each cell in the table reports the result of the pairwise comparison of the distribution of macroeconomic variables across different sizes, evaluated using an appropriate statistical test ${ }^{8}$. The null hypothesis of the Wilcoxon rank sum test is that the

\footnotetext{
7 See Wright (2005), p. 598, for the rationale behind doing so.

${ }^{8}$ For the less stringent case of $p=0.05$, a few combinations also exhibit size effects in addition to those in Table 3 . The variables and the corresponding combinations are: Worker Ratio (100/2000, 100/8000, 4000/10000, 8000/10000), Firm Demise Ratio
} 
two samples have identical medians and the null hypothesis of the K-S test is that the two distributions are identical. The check mark indicates that the null hypothesis is rejected for that cell, which lends support for the existence of the size effect. From Table 3, it is clear that most variables seem to exhibit size effects. The only exceptions are the worker ratios, the duration of the recession $(k)$, and firm growth distributions. The latter two seem to exhibit size effects for some ranges but are not consistent over all sizes.

Table 3: Significance results for size effects $-p=0.01$

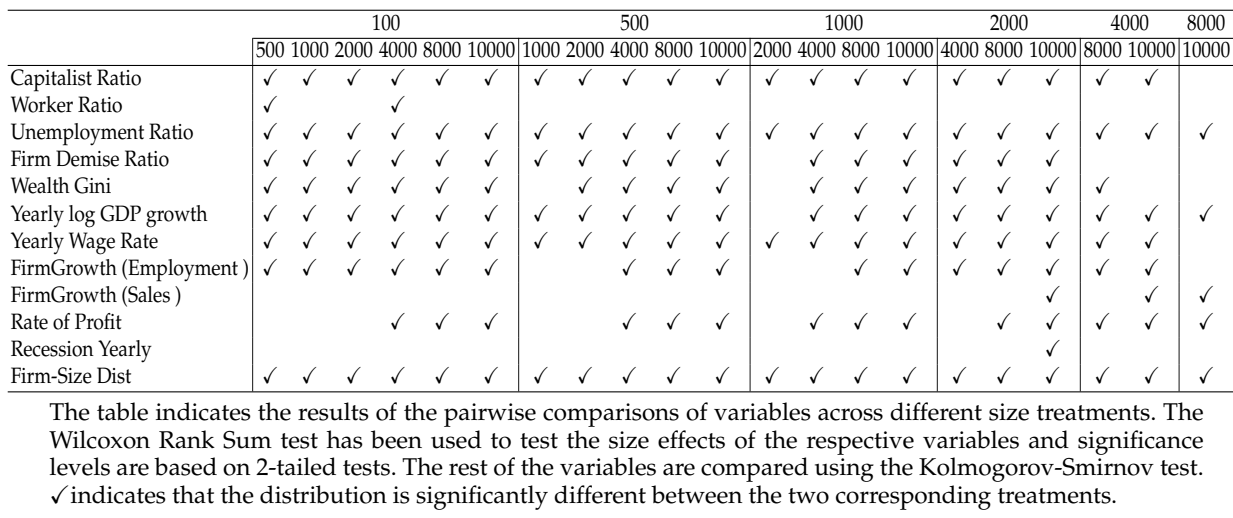

For variables involving class ratios such as the capitalist ratio, unemployment ratio, and worker ratio, we do observe a size effect. For the capitalist ratio, there is a trend of a declining mean (and the variance of this mean) as the size of the economy increases. For the unemployment ratio, the trend is the opposite and the mean ratio increases with size. There is no discernible trend in the worker's ratio, except for the fact that they are significantly different across sizes of the economy. The firm demise ratio exhibits a roughly declining trend when the size increases from a small value to a medium one. However, with over 4,000 agents the size effects seem to disappear. The wealth distribution is analysed across treatments by comparing the mean Gini co-efficients for the last 10 years (pooled for 100 repetitions) across different treatments. We find that the wealth distribution does exhibit size effects, but ceases to exist once the economy becomes large, indicating that the inequality tends to stabilize.

The distribution of yearly GDP growth rates evaluated using K-S tests indicates that there are significant size effects across treatments. The wage share distribution also indicates that there are significant size effects across treatments. Note that although the results of the K-S tests suggest that there are significant

(1000/2000), Wealth Gini (500/1000), Yearly Wage Share (8000/10000), Firm Growth (Employment) (500/1000, 500/2000), Rate of Profit (100/500, 100/2000, 500/2000, 2000/4000), and Recession Yearly (4000/10000). However, the overall patterns concerning the size effects remain unchanged. 
differences between the treatments, it is hard to conclude whether these differences are related only to the magnitude, or to the distribution, or to both.

The duration of the recession is calculated in retrospect after the simulation has been completed for 100 years. This variable by and large does not seem to exhibit size effects except for one or two cases. The firm growth distributions are examined for two different definitions of growth - in terms of sales and employment. In both cases, there are size effects, albeit for different, limited ranges. As for the employment-oriented definition, size effects are more evident. The rate of profit displays size effects, except in smaller economies. Firm size distribution, on the other hand, exhibits very clear size effects. Figure 4 illustrates the normalized firm size distributions for different agent size specifications.

Figure 3 demonstrates four aggregate variables that have demonstrated sizeeffects (for the median) throughout different treatments. The medians of the capitalist and unemployment ratios exhibit a decreasing and increasing trend with an increase in the size of the population, leaving worker ratios relatively static. The firm demise ratios exhibit a mild decreasing trend, although the magnitudes are negligible. The Gini coefficients of wealth distribution also exhibit a slightly increasing trend. One noticeable feature is that the variance (or the range of the data) decreases as the population of agents increases. We find that the smaller economies tend to have higher variances for the above specified variables and this could be because these variables are expressed in terms of ratios.

Some remarks are in order. When the K-S test does not reject the null hypothesis, we can be confident that both the distribution and the magnitude have no significance. However, in cases where the K-S test does reject the null hypothesis, we need to be more cautious about the conclusions that we draw. Consider two treatments resulting in different distributions, but with almost the same means. According to the K-S test, it is only suggested that one treatment makes the distribution more diverse or skewed.

\section{Conclusion}

We have examined the scale-free property of an agent-based macroeconomic model initially proposed by [13]. We find that many variables used in the model exhibit size effects. The issue of a size effect is more intricate than what we had expected. Some variables, especially those expressed in ratios, tend to stabilize with the systematic increase in the number of agents in our simulations, with a reduction in the spread of the means of these ratios. If such a stablization underpins a model, then it certainly becomes important to pay attention to size specifications. It is also worth mentioning that our mode of investigation may not be the only rigorous way of testing this size effect conjecture. We have adopted this approach to remain close to the analysis performed by Wright and to do so in the most intuitive way possible. Further research is required on this front. 


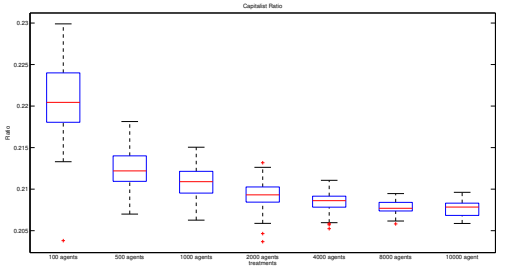

Capitalist ratio

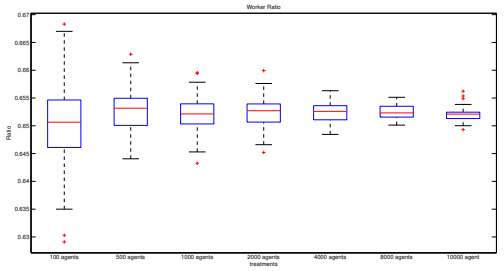

Worker ratio

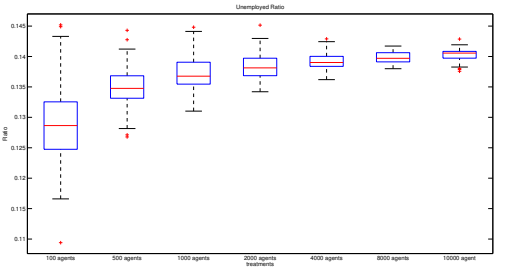

Unemployment ratio

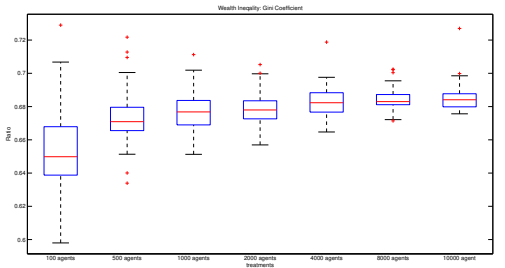

Wealth distribution (Gini coefficient)

Fig. 3: Size effects of different class and macroeconomic variables (capitalist ratio, unemployment ratio, worker ratio and wealth distribution (Gini coefficients)) are shown above. The deviations in their means across repetitions tend to become smaller as the sizes of the economies increase.
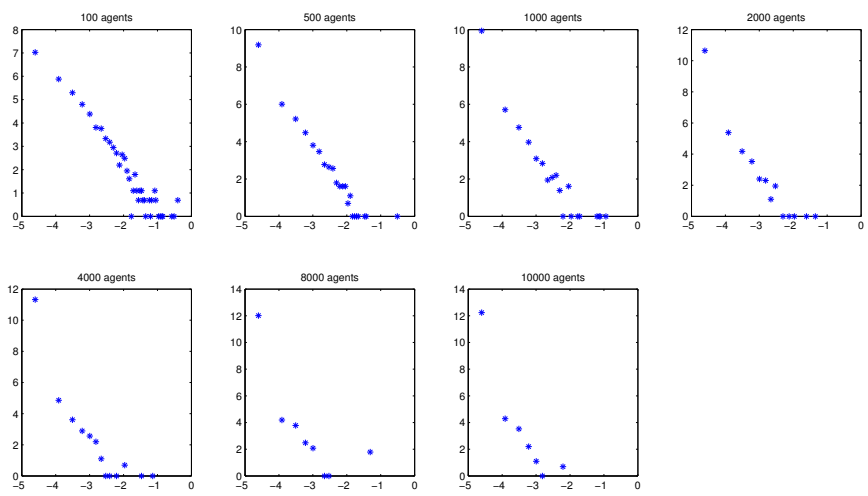

Fig. 4: Firm size distribution (normalized): The figures demonstrate the log-log plot of firm size distribution after normalizing it for the size of the economy since the absolute size of firms in the larger economy will be naturally bigger. The plots of smaller economies are visibly different from those of the larger economies. 
Although we find some initial evidence supporting size effects in this paper, further analysis is needed to unearth the possible mechanisms that make size a decisive variable. The appearance of the size effect for different variables may have unique causes for each of them and our interest, as a first step, has been to unearth common structures which generate this possibility. A comprehensive examination of the 'general' cause for the size effect requires an approach similar to 'big data analysis', which is beyond the scope of this paper. Our initial investigation can be seen as a first step to highlight the potential that underlies agent-based models to examine the possible roles of size in the macroeconomy.

\section{Acknowledgements}

The first and the last author are grateful for the research support in the form of the Ministry of Science and Technology (MOST) Grants, MOST 103-2410-H004-009-MY3 and MOST 104-2811-H-004-003, respectively.

\section{References}

1. Barabasi, A-L, Albert, R.: Emergence of scaling in random networks, Science 286:509512 (1999)

2. Challet D, Marsili M.: Criticality and market efficiency in a simple realistic model of the stock market, Physical Review 68(3): 036132-1-4 (2003)

3. Chen, S-H: Varieties of agents in agent-based computational economics: A historical and an interdisciplinary perspective, Journal of Economic Dynamics and Control 36(1):1-25 (2012)

4. Duffy, J., Unver, M.: Asset price bubbles and crashes with near zero-intelligence traders, Economic Theory 27:537-63(2006)

5. Egenter, E., Lux, T., Stauffer, D.: Finite-size effects in Monte Carlo simulations of two stock market models, Physica A: Statistical Mechanics and its Applications 268(1):250-256 (1999)

6. Farmer, D., Patelli, P., Zovko, I.: The predictive power of Zero Intelligence in financial markets, Proceedings of the National Academy of Sciences 102: 2254-2259 (2005)

7. Gode, D., Sunder, S.: Allocative efficiency of markets with zero intelligence traders: Market as a partial substitute for individual rationality, Journal of Political Economy, 101:119-137 (1993)

8. Lavicka H, Novotny J.: Employment, Production and Consumption with random update: Non-equilibrium stationary state equations, Acta Polytechnica, 53-6, 847-853 (2013)

9. Ladley, D: Zero intelligence in economics and finance, Knowledge Engineering Review 27(2): 273-286 (2012)

10. LeBaron, B., Tesfatsion, L.: Modeling macroeconomies as open-ended dynamic systems of interacting agents, American Economic Review, 98:2, 246-250 (2008)

11. Lux, T., Schornstein, S.: Genetic learning as an explanation of stylized facts of foreign exchange markets, Journal of Mathematical Economics 41(1-2):169-196 (2005)

12. Sunder, S.: Market as artifact: Aggregate efficiency from zero-intelligence traders, In Augier M, March J (eds.) Models of a Man: Essays in Memory of Herbert A. Simon, MIT Press, 501-519 (2004)

13. Wright, I.: The social architecture of capitalism, Physica A 346:589-620 (2005) 
14. Wright, I.: Implicit microfoundations for macroeconomics, Economics: The OpenAccess, Open-Assessment E-Journal 3:1-27 (2009). 